\title{
Diseño óptimo de sistemas de riego a presión Aplicación en casos (Ecuador)
}

\section{Optimal design of pressurized irrigation systems. Application cases (Ecuador)}

\section{Desenho ótimo de sistemas de irrigação por pressão Casos de aplicação (Equador)}

\author{
Carmen Mireya Lapo Pauta1 \& Rafael Pérez García² \\ 'Ingeniero Civil, Magister en Ingeniería Hidráulica y Medio Ambiente. 2Ingeniero Industrial, \\ Doctor en Ingeniería Hidráulica y Medio Ambiente ${ }^{1}$ Universidad Técnica Particular de Loja, \\ Ecuador. ${ }^{2}$ Instituto Universitario de Matemática Multidisciplinar, Departamento de Ingeniería \\ Hidráulica y Medio Ambiente, Universidad Politécnica de Valencia, España \\ ${ }^{1}$ cmlapo@utpl.edu.ec, ${ }^{2}$ rperez@gmmf.upv.es
}

\section{Resumen}

En el presente artículo, se presenta la investigación realizada con el fin de encontrar la mejor solución económica en el diseño de redes de riego a presión, y que a la vez se cumpla de forma eficiente la prestación de servicio. Se propone una metodología sistemática que combina dos técnicas de optimización a través de un "método híbrido", en el que se fusionan la programación lineal, la programación no lineal y los algoritmos genéticos. La formulación global del problema de dimensionado óptimo consistirá en minimizar una función objetivo, constituida por los costes asociados a las tuberías que configuran la red. Esta metodología se implementó en tres redes de riego, una ficticia y dos redes de riego (Tuncarta y Cariyacu) ubicadas en las ciudades de Loja y Chimborazo en donde se obtuvieron las soluciones de diseño óptimas. Finalmente se simularon diferentes escenarios en los dos modelos, para obtener una panorámica sobre el funcionamiento de las variables hidráulicas.

Palabras clave: criterio económico, emisor, métodos híbridos, turnos de riego, óptimo.

\section{Abstract}

This paper presents research completed with the intention of finding the most economical solution in the design of pressurized irrigation networks, while efficiently meet service delivery. A systematic methodology is proposed that combines two optimization techniques through a "hybrid method" in, which linear programming, nonlinear programming and genetic algorithms are fused. The overall formulations of the problem of optimal dimensioning consist of minimizing an objective function constituted through the asociated cost of the pipes that form the network. This methodology was implemented in three networks a fictitious irrigation and two irrigation networks (Tuncarta and Cariyacu) located in the cities of Loja and Chimborazo which yielded optimal design solutions. Finally different scenarios were simulated in both models to obtain an overview of the operation of the hydraulic variables

Key-words: economic criteria, emitter, hybrid methods, irrigation shifts, optimal. 


\section{Resumo}

Neste artigo, se apresenta a pesquisa realizada no intuito de encontrar a melhor solução econômica no desenho de redes de irrigação por pressão e o cumprimento de maneira eficiente à prestação do serviço. Propõe-se uma metodologia sistemática que combina as duas técnicas de eficiência através de um "método híbrido", no qual se fundem a programação linear, a programação não linear e os algoritmos genéticos. A formulação geral do problema de dimensionamento ótimo consistirá em minimizar uma função objetivo, constituída pelos custos associados com os tubos que conformam a rede. Esta metodologia foi inserida em três redes de irrigação, uma delas fictícia e duas (Tuncarta e Cariyacu) localizadas nas cidades de Loja e Chimborazo onde foram obtidas as soluções de desenho ideal. Finalmente diferentes cenários foram simulados em ambos os modelos para obter uma visão geral da operação das variáveis hidráulicas.

Palavras-chave: critério econômico, emissor, métodos híbridos, turnos de irrigação, ótimo

\section{Introducción}

La gestión sostenible, eficaz y equitativa de los recursos hídricos constituye un desafío clave para los próximos años, debido a que el consumo de agua ha aumentado en el doble debido al incremento de la población mundial durante el presente siglo. Considerando que el sector agrícola es uno de los ejes clave para el progreso del país (Ecuador) y que es un gran consumidor de este recurso, lo que se pretende en este trabajo es realizar un aporte para resolver los problemas de gestión de las redes de riego presurizadas.

En las actividades agrícolas, lo más usual en el medio geográfico donde se realizó la investigación, es la distribución del recurso hídrico a través de turnos de riego, razón por la cual se implementó una metodología para lograr que se cumplan parámetros de caudal y presión equilibrados según el requerimiento en los nudos demandantes; y además se buscó obtener el mínimo costo en el diseño de las líneas de tuberías, mediante la combinación óptima de hidrantes para cada turno que se encuentre asignado en los nudos, para lo cual se empleó una forma de optimización híbrida con Algoritmos Genéticos (AG), Programación Lineal y Programación No Lineal.

Las redes reales empleadas para la aplicación del modelo de optimización correspondieron a dos sectores de irrigación: 1) comunidad de Tuncarta, ubicado en la provincia de Loja, Cantón Saraguro, Parroquia Saraguro, con una altura promedio de 2.344 a 2.503 m.s.n.m.; el centro de la comunidad se ubica en las coordenadas 699200 E y 9567600 $\mathrm{N}$ aproximadamente. El sector de la red que se modeló está integrado por 41 hidrantes y 30 líneas, y se adoptaron tres turnos, con un tiempo total de riego de 4 horas por turno.

2) Comunidad de Cariyacu, perteneciente a la parroquia La Matriz, del cantón Patate, provincia de Tungurahua, la cual se halla ubicada en las coordenadas N 981137 E17 781137, a una altitud promedio de 2.400 m.s.n.m.; el sector modelado está conformado por 67 hidrantes y 49 líneas, se han considerado 2 turnos de 4 horas cada uno.

Varios autores han dedicado sus investigaciones a proponer métodos para que el diseño de redes presurizadas en abastecimiento o riego cumplan eficientemente las prestaciones de servicio y operación, considerando el factor económico como una variable decisiva en la resolución de este tipo de problemas. Calvo et al. (2009), trabajaron en un algoritmo que combina programación dinámica y el cálculo hidráulico de redes presurizadas para organizar los turnos de una jornada de riego determinada, consiguiendo minimizar el consumo de la energía que demanda la red de riego en todos los turnos. Lara (2007), desarrolló un modelo de diseño 
óptimo de redes colectivas de riego que incluye y aprovecha de forma eficiente la distribución de caudales por turnos en donde se logra un equilibrio energético en los mismos, cuando se trata de una red alimentada por sistema de bombeo. Por su parte, Mora et al. (2010) minimizaron costes de inversión de redes de abastecimiento a través de técnicas evolutivas obteniendo resultados satisfactorios, los cuales se adoptan en función de las necesidades de diseño en cada caso particular. Villa (2010) propone una metodología para la ubicación óptima de hidrantes en una red de riego a través de un modelo de programación entera binaria que obtiene eficiencia espacial. En particular en este trabajo se van a implementar los modelos usando Programación Lineal, Programación No Lineal y Algoritmos Genéticos en redes de riego a presión, como ya se había mencionado.

El objetivo general de la investigación fue desarrollar una metodología para el diseño óptimo de redes abiertas de riego a presión funcionando por turnos. Y, como objetivos específicos se consideraron: 1) plantear una secuencia lógica para encontrar el costo mínimo en diámetros de tuberías, a través de la combinación óptima de hidrantes para cada turno que se encuentre asignado en nudos demandantes; 2) Implementar modelos usando programación lineal, programación no lineal y algoritmos genéticos; $y$, 3) contrastar resultados de los modelos desarrollados en las redes de riego empleadas.

\section{Materiales y métodos}

- El soporte informático que se usó en el presente trabajo se enumera a continuación:

- Software Diopram 3.0, versión PREMIUM, desarrollado por el equipo multidisciplinario de Mecánica de Fluidos de la Universidad Politécnica de Valencia.

- Software Epanet 2.0 versión libre, que desarrolló la EPA (United States Environmental Protection Agency)
- Software Evolver Window versión reléase 4.0, Palisade Corporation.

- Microsoft Excel, aplicación Solver.

Para realizar la modelación del método híbrido desarrollado se usaron datos de tres redes de riego: una ficticia y dos redes que corresponden a comunidades regantes ubicadas en las ciudades de Loja y Chimborazo que son Tucarta y Cariyacu.

\section{Protocolo implementado}

La secuencia que se sigue para el desarrollo de los dos modelos es como sigue: Método Plyag

En el método híbrido (PLYAG) (Baños, 2006), se combina la programación lineal $\mathrm{PL}$, que ha sido abordada por diversos autores como: Labye (1966); Karmeli et al. (1968); Calhoun (1970), y en la misma línea Bhave (1979); y algoritmos genéticos (AG).

La secuencia de desarrollo es como sigue:

1. Definición del trazado de la red, turnos de riego, ubicación de hidrantes, cálculo del caudal de diseño por línea que resulta de la sumatoria de caudales de las tomas o hidrantes abiertos aguas abajo en cada turno de riego.

2. Cálculo de la probabilidad de funcionamiento de los hidrantes en los turnos designados, Lamaddalena y Sagardoy (2000).

$$
\mathrm{p}=\frac{\mathrm{q}_{\mathrm{f}} \cdot \mathrm{S}}{\mathrm{q}_{\mathrm{D}}} * \mathrm{~K}
$$

Donde: S: superficie servida en ha; qf: caudal ficticio continuo $\mathrm{l} / \mathrm{s} ; \mathrm{q}_{\mathrm{D}}$ : dotación suministrada por hidrante en $\mathrm{I} / \mathrm{s}$.

3. Ingresar topología de la red.

4. Aplicación del modelo de programación lineal definiendo la función objetivo y las restricciones 
para minimizar costo. Como hipótesis de diseño se consideran múltiples estados de carga, (n turnos), con una única fuente de alimentación y altura piezométrica conocida.

La formulación global del problema de dimensionado óptimo, Alperovits y Shamir (1977) consistirá en minimizar la función objetivo, configurada por los costes asociados a las tuberías resolución (Pérez, 1993), esto es:

$$
C_{i}=\sum_{i=1}^{k} \sum_{j=1}^{N D} c_{j} \cdot L_{i, j}
$$

Donde:

Ci: Coste total de las tuberías de la línea i (€); Cj: Coste unitario del diámetro $D$ (€/metro lineal); Lij: Longitud del tramo de diámetro $D$ en la línea i $(m)$.

Se consideran las restricciones:

- Presión mínima en cada turno 1, 2, o 3:

$$
\sum_{i \in S_{0 m k}} h_{f,}=\sum_{i \in S_{0, m}} \sum_{j=1}^{N D} j_{i, j k} \cdot L_{i, j} \leq \Delta H_{0, m, k} \forall \text { turnokynudom }
$$

\section{Donde:}

Ji,j,kLi,j.- Pérdida de carga (m) en la línea i, del turno k; So,m,k.- Conjunto de líneas pertenecientes al trayecto entre los nudos 0 y $\mathrm{m}$ con su respectivo turno k; $\Delta \mathrm{Ho}, \mathrm{m}, \mathrm{k}$.- Máxima pérdida de carga admisible $(m)$ en el trayecto comprendido entre los nudos 0 y $\mathrm{m}$ y el turno $\mathrm{k}$ que corresponda.

Limitaciones de tipo geométrico, a fin de asegurar que la suma de las longitudes parciales Lij, en una línea sea exactamente igual a la longitud de la misma:

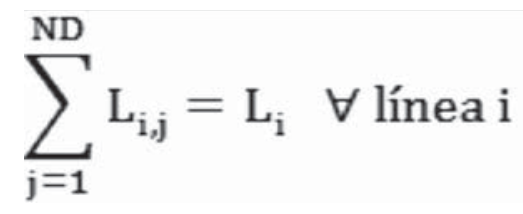

[4]

Delimitar las ventanas de diámetros candidatos por limitación de velocidad, con el fin de evitar los problemas de sedimentación y erosión, en el rango de $[0,50 ; 2,00] \mathrm{m} / \mathrm{s}$, obteniendo una matriz que resulta de superponer los rangos de diámetros que se obtienen en cada línea para cada turno de riego definido, de manera que el problema queda mucho más acotado, Bhave (1979) plantea que el conjunto óptimo de diámetros candidatos para la línea i, puede tener dos diámetros normalizados consecutivos en tamaño.

Ensamblar el modelo de asignación de turnos, para lo cual se usarán los datos de: cantidad de hidrantes, número de nudo, número de hidrante, número de turno, área de cobertura de riego, caudal demandado.

Combinar hidrantes para optimizar el costo total a través del software Evolver, en el que inicialmente se plantean turnos al azar en los nudos con hidrantes de la red; se ejecuta la macro denominada Solviendo, en la cual se encuentran establecidas las restricciones de partida. El coste de la solución óptima de cada una de las combinaciones de turnos constituye el valor asociado a las soluciones de Evolver, que determina el subsiguiente proceso de reproducción dentro del Algoritmo Genético, iniciándose un proceso iterativo hasta encontrar la solución deseada. El diagrama de flujo que corresponde a la optimización por el método híbrido planteado se muestra en la Figura 1. 


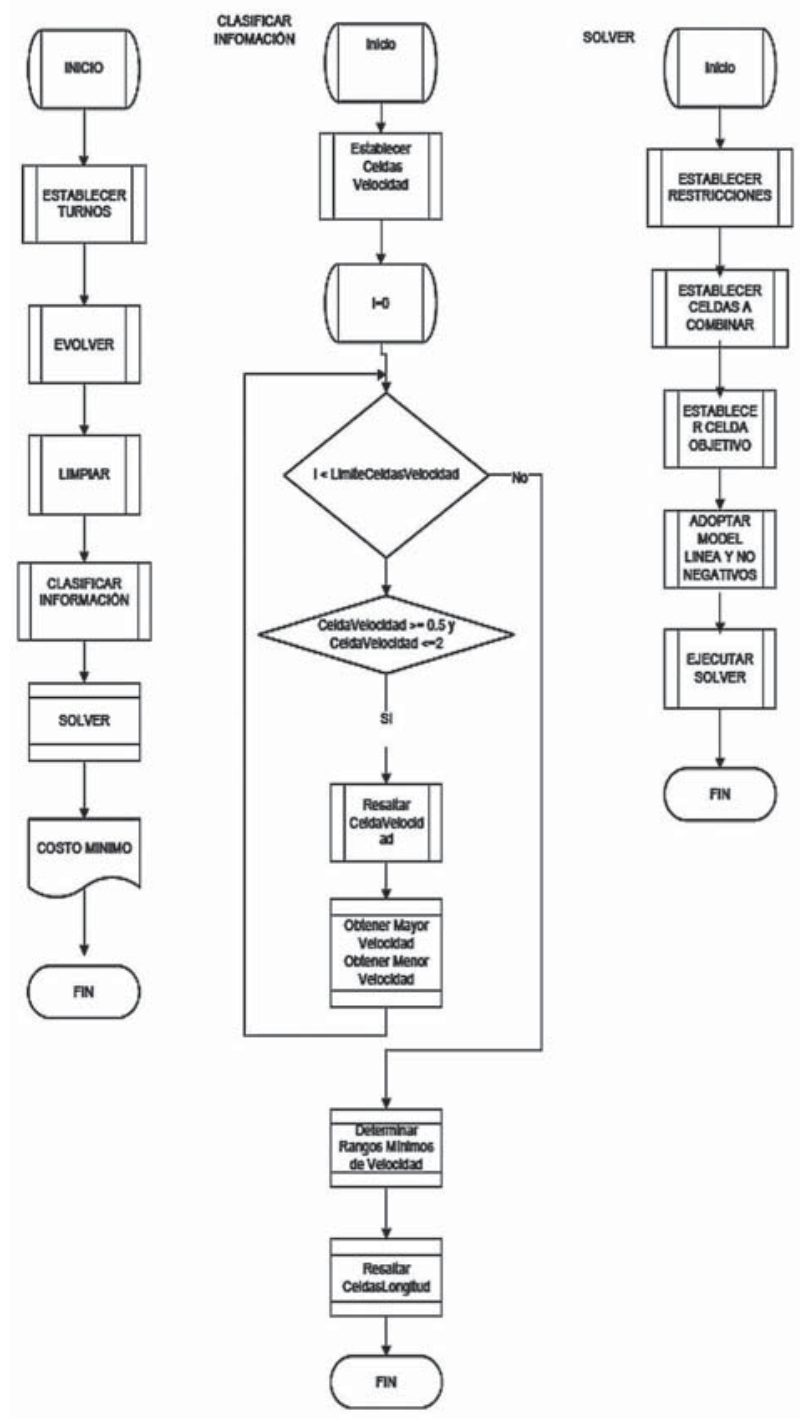

Figura 1. Diagrama de flujo correspondiente a la optimización por el método híbrido PLYAG

\section{Método PNLYAG}

Consiste en el desarrollo con Programación No Lineal (PNL) y Algoritmos Genéticos (AG) De Jong (1975) y Davies y Príncipe (1991), la secuencia para implementar el modelo se detalla:

1. Aplicación de los ítems 1, 2, 3 del modelo híbrido (PLYAG).

2. Aplicación del modelo de Programación No Lineal, definiendo la función objetivo y las restricciones con el fin de minimizar el costo.
Se ha estimado un conjunto de estados de carga, correspondiente a $\mathrm{n}$ turnos, con una fuente de alimentación con altura piezométrica conocida como hipótesis de diseño.

La función objetivo, corresponde:

$$
C_{i}=\sum_{i=1}^{k} \sum_{j=1}^{N D} c_{j} \cdot L_{i, j}
$$

El coste por metro lineal de las tuberías empleadas resulta de la ecuación procedente del ajuste de los costes de los diámetros comerciales discretos mediante una relación potencial del tipo $c=A \cdot D^{b}$,paralaseriepropuestaenlas redes de estudio es: $c=0,0011929 \cdot D^{1,9434121}$

\section{Restricciones}

- Las restricciones de presión mínima en cada turno 1, 2, o 3 son:

$$
\sum_{k=0, m, k} h_{f, i}=\sum_{i 0_{0, m}} \sum_{j=1}^{N D} j_{i, j, k} \cdot L_{i, j} \leq \Delta H_{0, m, k} \forall \text { turno } k \text { y nudo } m
$$

- Limitaciones de diámetros mínimos y máximos que se deben considerar en la red,

$$
m m<d<1000 \mathrm{~mm} \quad[7]
$$

3. Ensamblado del modelo de asignación de turnos, similar al literal 6, de la metodología del modelo híbrido PLYAG.

4. Combinación de hidrantes para optimizar el costo total similar al ítem 7 de la metodología del modelo híbrido PLYAG.

El diagrama de flujo que refleja el procedimiento del modelo hibrido PNLYAG es presentado en la Figura 2. 


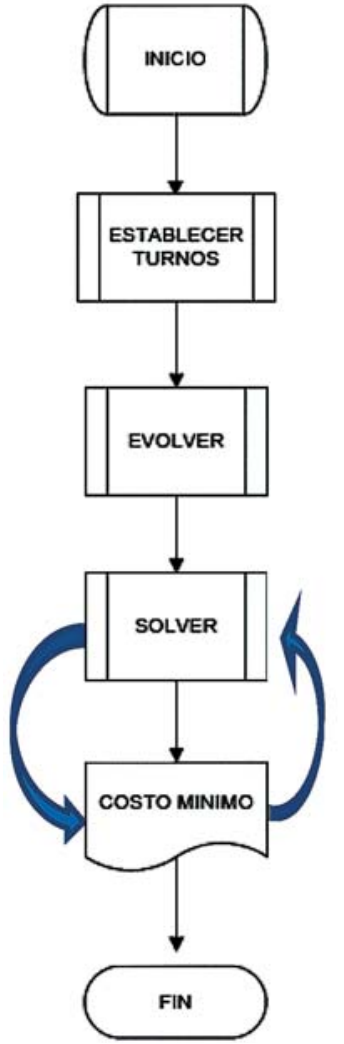

Figura 2. Diagrama de flujo correspondiente a la optimización por el método híbrido PNLYAG

\section{Resultados y discusión}

En la red número 1 que consta de 4 líneas con 10 hidrantes, se aplicó el método PLYAG cuyos resultados demostraron la bondad del método y se obtuvo el diseño óptimo buscado.

\section{Sistema Uno}

El sistema de riego que se asume consta de tres turnos:

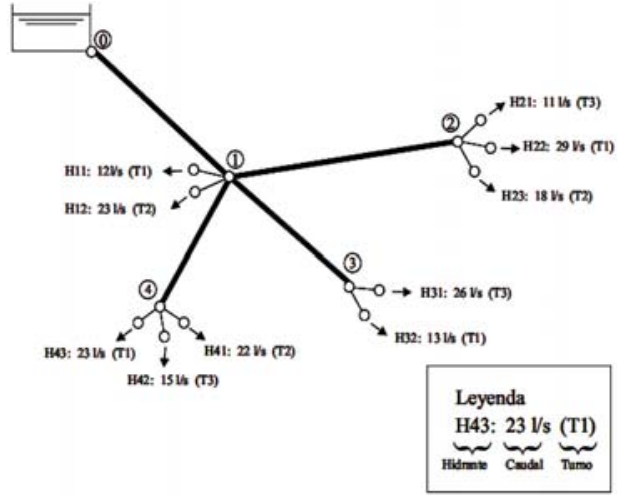

Los caudales que corresponden a cada turno:
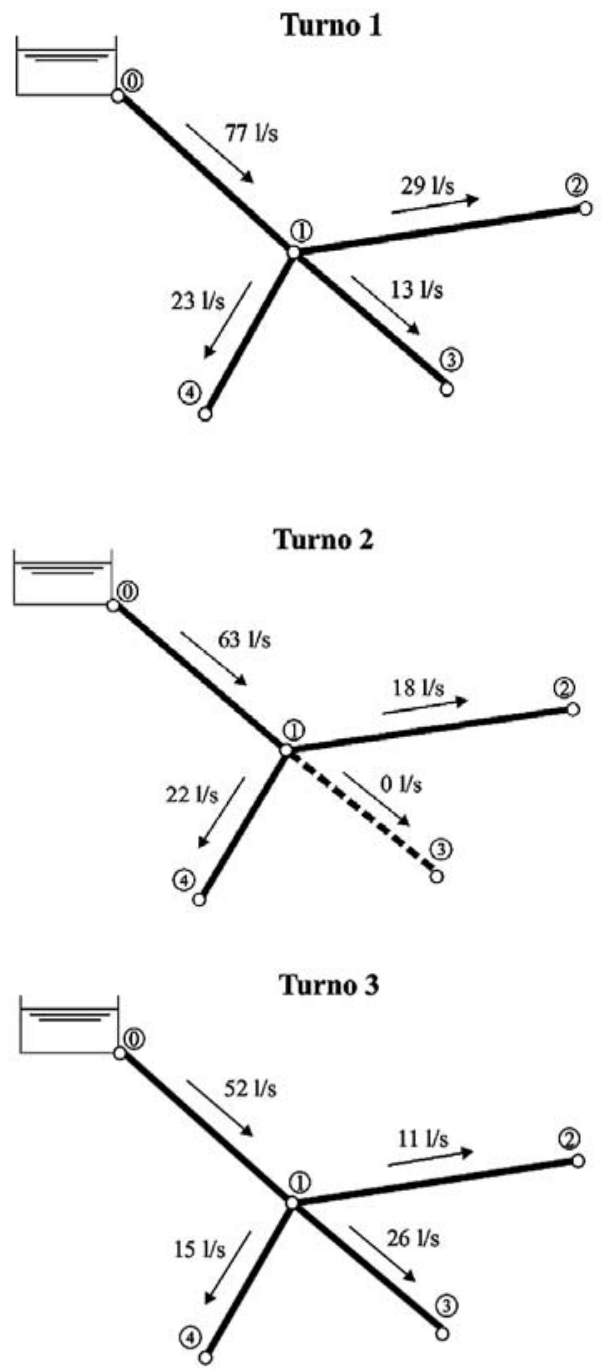

Figura 3. Caudales circulantes en los turnos de la red uno.

Los parámetros iniciales asumidos son mostrados en la Tabla 1:

Tabla. 1. Parámetros iniciales:

\begin{tabular}{lcc} 
Viscosidad cinemática & 0.000001 & $\mathrm{~m} / \mathrm{s}$ \\
\hline Rugosidad & 0.01 & $\mathrm{~mm}$ \\
\hline Caudal ficticio continuo & 1 & $\mathrm{l} /(\mathrm{s} . \mathrm{ha})$ \\
\hline Jornada de riego & 12 & horas \\
\hline Número de turnos & 3 & \\
\hline
\end{tabular}


El modelo de la red 1, se observa en la (Tabla 2):

Tabla 2. Datos del modelo:

\begin{tabular}{|c|c|c|c|}
\hline NH & Nudo & $\begin{array}{l}\text { Hidrante } \\
\text { (h) }\end{array}$ & $\begin{array}{l}\text { Área x h } \\
\text { (ha) }\end{array}$ \\
\hline 1 & 1 & 1 & 2'652 \\
\hline 2 & 1 & 2 & 4'448 \\
\hline 3 & 2 & 1 & 6'948 \\
\hline 4 & 2 & 2 & 4'229 \\
\hline 5 & 2 & 3 & 2'466 \\
\hline 6 & 3 & 1 & 2'933 \\
\hline 7 & 3 & 2 & 5'895 \\
\hline 8 & 4 & 1 & 4'679 \\
\hline 9 & 4 & 2 & 4'766 \\
\hline 10 & 4 & 3 & 3'130 \\
\hline
\end{tabular}

Los caudales circulantes resultantes en cada una de las líneas de la red corresponden a lo mostrado en la Tabla 3.

En el modelo PLYAG se toma como una de las restricciones el rango de velocidad $(0,5$ a $2 \mathrm{~m} / \mathrm{s})$, y considerando para el diseño la serie de diámetros de tuberías (existentes en Ecuador). Se obtuvieron las ventanas de diámetros candidatos por limitaciones de velocidad, de manera que el problema queda mucho más acotado y el número de condiciones y restricciones necesarias para resolverlo disminuye consecuentemente Bhave (1979). Se observa en la Tabla 4, el resultado de diámetros candidatos que cumplen con el rango de velocidad.
Tabla 3. Caudales circulantes según turno.

Turno 1

\begin{tabular}{cccc}
\hline $\begin{array}{c}\text { Línea I } \\
\text { Nudo }\end{array}$ & Cota (m) & $\begin{array}{c}\text { Caudal } \\
\text { línea (I/s) }\end{array}$ & $\begin{array}{c}\text { Longitud } \\
(\mathbf{m})\end{array}$ \\
\hline 0 & 80 & & \\
\hline 1 & 40 & 77 & 2.000 \\
\hline 2 & 30 & 29 & 2.400 \\
\hline 3 & 45 & 13 & 1.200 \\
\hline 4 & 20 & 23 & 1.500 \\
\hline
\end{tabular}

Turno 2

\begin{tabular}{cccc}
$\begin{array}{c}\text { Línea / } \\
\text { Nudo }\end{array}$ & $\begin{array}{c}\text { Cota } \\
(\mathbf{m})\end{array}$ & $\begin{array}{c}\text { Caudal } \\
\text { línea (I/s) }\end{array}$ & $\begin{array}{c}\text { Longitud } \\
(\mathrm{m})\end{array}$ \\
\hline 0 & 80 & & \\
\hline 1 & 40 & 63 & 2.000 \\
\hline 2 & 30 & 18 & 2.400 \\
\hline 3 & 45 & 0 & 1.200 \\
4 & 20 & 22 & 1.500 \\
\hline
\end{tabular}

Turno 3

\begin{tabular}{cccc}
$\begin{array}{c}\text { Línea I } \\
\text { Nudo }\end{array}$ & Cota (m) & $\begin{array}{c}\text { Caudal } \\
\text { línea (I/s) }\end{array}$ & $\begin{array}{c}\text { Longitud } \\
(\mathbf{m})\end{array}$ \\
\hline 0 & 80 & & \\
\hline 1 & 40 & 52 & 2.000 \\
\hline 2 & 30 & 11 & 2.400 \\
\hline 3 & 45 & 26 & 1.200 \\
4 & 20 & 15 & 1.500 \\
\hline
\end{tabular}




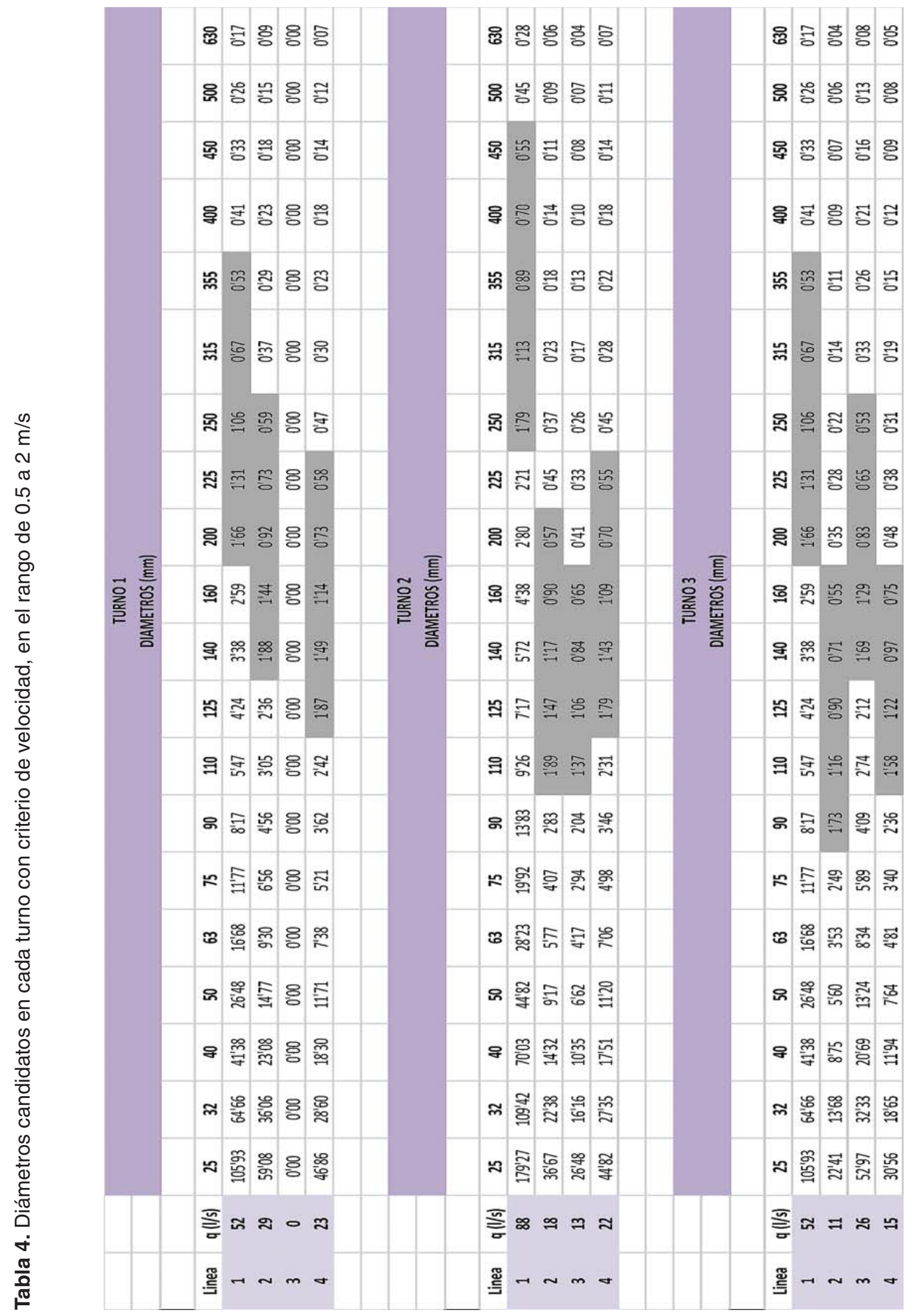


Luego de acotar los valores de velocidad, se implementa el modelo de programación lineal Pérez, R. (1993) en la red 1, y se obtiene la longitud de tubería que corresponde a cada diámetro en las líneas de la red, sus resultados se observan en la Tabla 5.

Tabla 5. Valores de longitud en cada línea del modelo, luego de aplicar PL

\begin{tabular}{|c|c|c|c|c|c|c|c|}
\hline \multirow[b]{2}{*}{ Linea } & \multirow[b]{2}{*}{$q(1 / s)$} & \multicolumn{6}{|c|}{ DIAMETROS (mm) } \\
\hline & & $140^{\prime} 00$ & $160^{\prime} 00$ & $200^{\prime} 00$ & $225^{\prime} 00$ & $250^{\prime} 00$ & $315^{\prime} 00$ \\
\hline $1^{\prime} 00$ & $52^{\prime} 00$ & $0^{\prime} 00$ & $0^{\prime} 00$ & $0^{\prime} 00$ & $0^{\prime} 00$ & $1.849^{\prime} 80$ & $150^{\prime} 20$ \\
\hline $2^{\prime} 00$ & $11^{\prime} 00$ & $0^{\prime} 00$ & $1.783^{\prime} 88$ & $616^{\prime} 12$ & $0^{\prime} 00$ & $0^{\prime} 00$ & $0^{\prime} 00$ \\
\hline $3^{\prime} 00$ & $26^{\prime} 00$ & $0^{\prime} 00$ & $995^{\prime} 48$ & $204^{\prime} 52$ & $0^{\prime} 00$ & $0^{\prime} 00$ & $0^{\prime} 00$ \\
\hline $4^{\prime} 00$ & $15^{\prime} 00$ & $1.200^{\prime} 76$ & $299^{\prime} 24$ & $0^{\prime} 00$ & $0^{\prime} 00$ & $0^{\prime} 00$ & $0^{\prime} 00$ \\
\hline
\end{tabular}

El costo total por tramos en cada una de las líneas de la red, se muestra en la tabla 6.

Tabla 6. Costes totales de las líneas de la red

\begin{tabular}{|c|c|c|c|c|c|c|c|c|}
\hline & & \multicolumn{7}{|c|}{ DIAMETROS (mm) } \\
\hline Linea & $q(\mathbf{l} / \mathbf{s})$ & $140^{\prime} 00$ & $160^{\prime} 00$ & $200^{\prime} 00$ & $225^{\prime} 00$ & $250^{\prime} 00$ & $315^{\prime} 00$ & Costo \\
\hline $1^{\prime} 00$ & $77^{\prime} 00$ & $0^{\prime} 00$ & $0^{\prime} 00$ & $0^{\prime} 00$ & $0^{\prime} 00$ & $100.905^{\prime} 56$ & $12.838^{\prime} 46$ & $113.744^{\prime} 02$ \\
\hline $2^{\prime} 00$ & $29^{\prime} 00$ & $0^{\prime} 00$ & $40.877^{\prime} 46$ & $21.782^{\prime} 95$ & $0^{\prime} 00$ & $0^{\prime} 00$ & $0^{\prime} 00$ & $62.660^{\prime} 41$ \\
\hline $3^{\prime} 00$ & $13^{\prime} 00$ & $0^{\prime} 00$ & $22.811^{\prime} 25$ & $7.230^{\prime} 93$ & $0^{\prime} 00$ & $0^{\prime} 00$ & $0^{\prime} 00$ & $30.042^{\prime} 18$ \\
\hline $4^{\prime} 00$ & $23^{\prime} 00$ & $21.226^{\prime} 20$ & $6.857^{\prime} 00$ & $0^{\prime} 00$ & $0^{\prime} 00$ & $0^{\prime} 00$ & $0^{\prime} 00$ & $28.083^{\prime} 20$ \\
\hline & & & & & & & & $234.529^{\prime} 80$ \\
\hline
\end{tabular}

La altura de energía de la red se observa en la Tabla 7, verificándose que la condición de presión requerida se cumple satisfactoriamente, en las líneas que corresponden a cada turno. 
Tabla 7. Presión mínima en los nudos demandantes:

\begin{tabular}{|c|c|c|c|c|}
\hline Zo & 80 & $\mathrm{~m}$ & & \\
\hline \multicolumn{5}{|l|}{ TURNO 1} \\
\hline PERDIDAS & PERD AL NUDO & $z$ & pmin & Zo-Hmin \\
\hline $8^{\prime} 68$ & $8^{\prime} 68$ & 40 & $15^{\prime} 00$ & $25^{\prime} 00$ \\
\hline $26^{\prime} 32$ & $35^{\prime} 00$ & 30 & $15^{\prime} 00$ & $35^{\prime} 00$ \\
\hline $0^{\prime} 00$ & $8^{\prime} 68$ & 45 & $15^{\prime} 00$ & $20^{\prime} 00$ \\
\hline $22^{\prime} 01$ & $30^{\prime} 69$ & 20 & $15^{\prime} 00$ & $45^{\prime} 00$ \\
\hline \multicolumn{5}{|l|}{ TURNO 2} \\
\hline $24^{\prime} 86$ & $24 ' 86$ & 40 & $15^{\prime} 00$ & $25^{\prime} 00$ \\
\hline $10^{\prime} 14$ & $35^{\prime} 00$ & 30 & $15^{\prime} 00$ & $35^{\prime} 00$ \\
\hline $2^{\prime} 83$ & $27^{\prime} 69$ & 45 & $0^{\prime} 00$ & $35^{\prime} 00$ \\
\hline $20^{\prime} 14$ & $45^{\prime} 00$ & 20 & $15^{\prime} 00$ & $45^{\prime} 00$ \\
\hline \multicolumn{5}{|l|}{ TURNO 3} \\
\hline $8^{\prime} 68$ & $8^{\prime} 68$ & 40 & $15^{\prime} 00$ & $25^{\prime} 00$ \\
\hline $3^{\prime} 79$ & $12^{\prime} 47$ & 30 & $15^{\prime} 00$ & $35^{\prime} 00$ \\
\hline 11'32 & $20^{\prime} 00$ & 45 & $15^{\prime} 00$ & $20^{\prime} 00$ \\
\hline $9^{\prime} 36$ & $18^{\prime} 04$ & 20 & $15^{\prime} 00$ & $45^{\prime} 00$ \\
\hline
\end{tabular}

El resultado de la combinación óptima de hidrantes luego de aplicar el modelo híbrido PLYG en la red uno, se observa en la Tabla 8:

Tabla 8. Combinación óptima de hidrantes y coste total en la red uno

\begin{tabular}{|c|c|c|c|c|c|}
\hline $\mathbf{N H}$ & Nudo & Hidran & TURNO & Ah (ha) & Qh (1/s) \\
\hline 1 & 1 & 1 & 2 & $2^{\prime} 652$ & $12^{\prime} 0$ \\
\hline 2 & 1 & 2 & 2 & $4^{\prime} 448$ & $23^{\prime} 0$ \\
\hline 3 & 2 & 1 & 1 & 6'948 & $29^{\prime} 0$ \\
\hline 4 & $\underline{2}$ & 2 & 2 & $4^{\prime} 229$ & $18^{\prime} 0$ \\
\hline 5 & 2 & 3 & 3 & $2^{\prime} 466$ & $11^{\prime} 0$ \\
\hline 6 & $\underline{3}$ & 1 & 2 & 2'933 & $13^{\prime} 0$ \\
\hline 7 & 3 & 2 & 3 & 5'895 & $26^{\prime} 0$ \\
\hline 8 & 4 & 1 & 1 & $4^{\prime} 679$ & $23^{\prime} 0$ \\
\hline 9 & 4 & 2 & 2 & $4^{\prime} 766$ & $22^{\prime} 0$ \\
\hline 10 & 4 & 3 & 3 & $3^{\prime} 130$ & $15^{\prime} 0$ \\
\hline
\end{tabular}

\begin{tabular}{|ll|l|}
\hline Coste $234,529 ' 80$ & USD \\
\hline
\end{tabular}

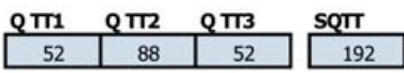

QT1
\begin{tabular}{|c|c|c|}
\hline Q T2 & Q T3 \\
\hline 0 & 12 & 0 \\
\hline 0 & 23 & 0 \\
\hline 29 & 0 & 0 \\
\hline 0 & 18 & 0 \\
\hline 0 & 0 & 11 \\
\hline 0 & 13 & 0 \\
\hline 0 & 0 & 26 \\
\hline 23 & 0 & 0 \\
\hline 0 & 22 & 0 \\
\hline 0 & 0 & 15 \\
\hline
\end{tabular}

Se constata que los resultados que se obtienen al usar el método PLYAG, constituyen el 50\% del costo usando el software Diopram con la red trabajando en la modalidad a la demanda, como se observa en la Tabla 9:

Tabla 9. Resultados obtenidos al usar el método PLYAG

\begin{tabular}{cccccc}
\hline Líneas & Caudal & Longitud & Diámetro & $\begin{array}{c}\text { Coste } \\
\text { /M }\end{array}$ & $\begin{array}{c}\text { Coste } \\
\text { Total }\end{array}$ \\
\hline 1 & 123,471 & 2000 & 450 & 116,61 & 233.220 \\
2 & 56,97 & 2400 & 225 & 29,97 & 71.928 \\
3 & 42,818 & 1200 & 315 & 62,09 & 74.508 \\
4 & 53,488 & 1500 & 355 & 110,8 & 166.200 \\
& & & & & $\$ 545.856$ \\
\hline
\end{tabular}

\section{Sistema Dos (Tuncarta)}

El sector de la red que se modeló está integrado por 41 hidrantes y 30 líneas, y se adoptaron tres turnos, con un tiempo total de riego de 4 horas por turno.

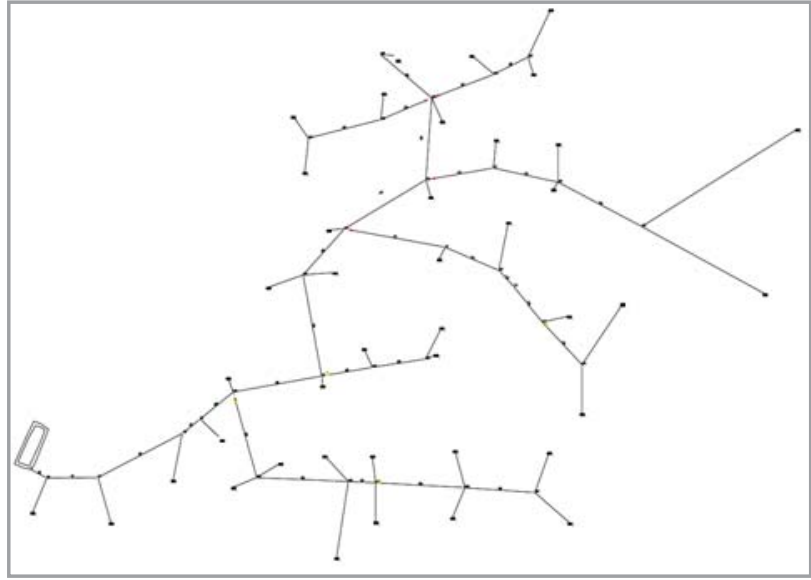

Figura 4. Líneas que constituyen el sistema de riego Tuncarta

La relación obtenida para la serie propuesta en la red que nos atañe es: $c=0,0011929 \cdot D^{1{ }^{1943412}}$, que se obtiene de los costos de tubería en la región. 


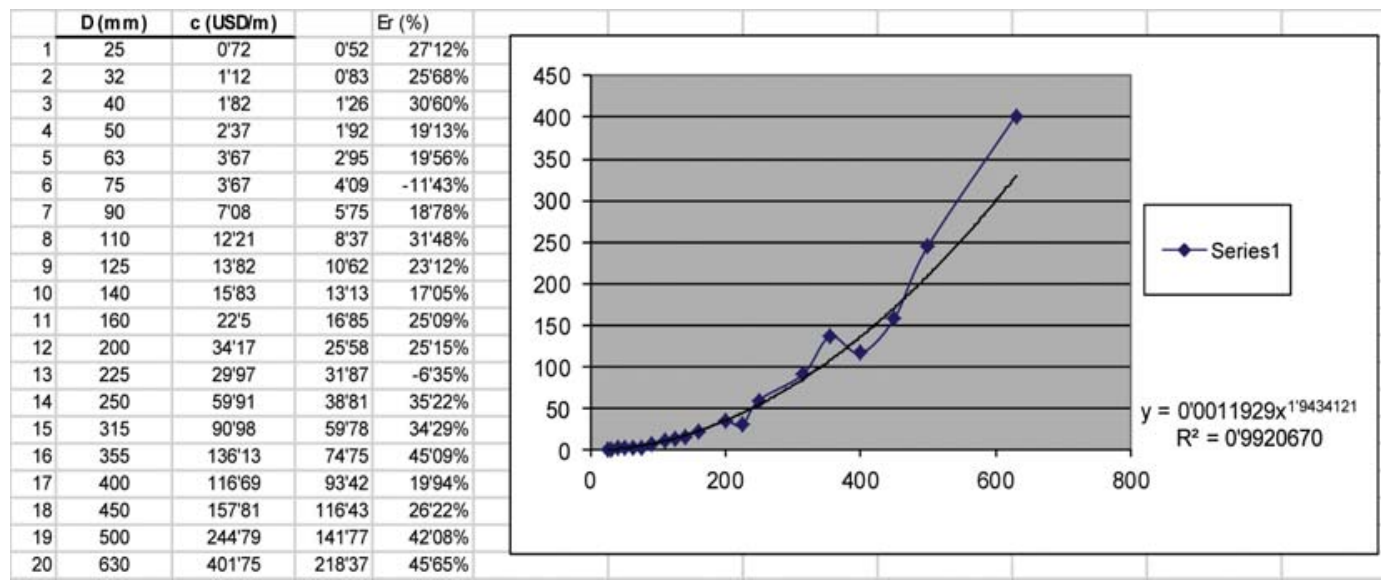

Figura 5. Relación Diámetro-Costos

La combinación de hidrantes resultante es la que se puede apreciar en color rojo en la tabla 10:

Tabla 10. Combinación de hidrantes

\begin{tabular}{|c|c|c|c|c|c|c|c|c|c|c|c|}
\hline $\mathrm{NH}$ & Nudo & Hidrante & $\begin{array}{c}\text { TUR- } \\
\text { NO }\end{array}$ & $\begin{array}{l}\text { Ah } \\
\text { (ha) }\end{array}$ & $\begin{array}{c}\text { Qh } \\
\text { (I/s) }\end{array}$ & NH & Nudo & Hidrante & $\begin{array}{c}\text { TUR- } \\
\text { NO }\end{array}$ & $\begin{array}{c}\text { Ah } \\
\text { (ha) }\end{array}$ & $\begin{array}{c}\text { Qh } \\
\text { (I/s) }\end{array}$ \\
\hline 1 & 96 & 1 & 3 & 0'177 & 0'184 & 22 & 110 & 1 & 1 & 0'543 & 0'565 \\
\hline 2 & 97 & 1 & 2 & 0'974 & 1'013 & 23 & 111 & 1 & 1 & 0’946 & 0'984 \\
\hline 3 & 98 & 1 & 2 & 0'317 & 0’330 & 24 & 112 & 1 & 2 & 0’599 & 0,623 \\
\hline 4 & 99 & 1 & 1 & 4'937 & $5 ’ 134$ & 25 & 114 & 1 & 2 & 0’365 & 0,379 \\
\hline 5 & 100 & 1 & 3 & 0’544 & 0’566 & 26 & \multirow{2}{*}{115} & 1 & 1 & 2'952 & 3 '070 \\
\hline 6 & 101 & 1 & 2 & 0'197 & 0’205 & 27 & & 2 & 3 & 2’952 & $3 ’ 070$ \\
\hline 7 & 102 & 1 & 2 & 0’729 & 0’759 & 28 & 116 & 1 & 1 & $1 ' 148$ & 1'194 \\
\hline 8 & \multirow{2}{*}{103} & 1 & 2 & 1'610 & 1'674 & 29 & 117 & 1 & 2 & 0’808 & 0'840 \\
\hline 9 & & 2 & 3 & 1'610 & $1^{\prime} 674$ & 30 & \multirow[b]{2}{*}{118} & 1 & 3 & 0’584 & 0,608 \\
\hline 10 & \multirow{2}{*}{104} & 1 & 1 & 0’956 & 0'994 & 31 & & 2 & 1 & 0’584 & 0,608 \\
\hline 11 & & 2 & 2 & 0’956 & 0’994 & 32 & \multirow{2}{*}{119} & 1 & 2 & 5'513 & $5^{\prime} 733$ \\
\hline 12 & 105 & 1 & 1 & 2'054 & $\begin{array}{l}\text { 2'136 } \\
\text { '136 }\end{array}$ & & & 2 & 3 & $5 ' 513$ & $5^{\prime} 733$ \\
\hline 14 & \multirow[b]{2}{*}{106} & $\begin{array}{l}2 \\
1\end{array}$ & 2 & $1 ' 224$ & $1 ' 273$ & \multirow{2}{*}{35} & 120 & 1 & 1 & 0’339 & 0,353 \\
\hline 15 & & 2 & 2 & 1'224 & $1 ' 273$ & & 121 & 1 & 2 & 0’844 & 0'877 \\
\hline 16 & \multirow{2}{*}{107} & 1 & 2 & 0'778 & 0’809 & \multirow{2}{*}{$\begin{array}{l}36 \\
37\end{array}$} & \multirow{2}{*}{122} & 1 & 3 & 1’054 & 1'096 \\
\hline 17 & & 2 & 2 & $0 ' 778$ & 0’809 & & & 2 & 2 & 1'054 & $1 ' 096$ \\
\hline 18 & \multirow{2}{*}{108} & 1 & 3 & 0'844 & 0'878 & 38 & 123 & 1 & 1 & 0’466 & 0'485 \\
\hline 19 & & 2 & 2 & 0'844 & 0’878 & 39 & \multirow{2}{*}{124} & 1 & 1 & 0’549 & 0'571 \\
\hline 20 & \multirow{2}{*}{109} & 1 & 1 & 3'738 & 3’888 & 40 & & 2 & 3 & 0’549 & 0’571 \\
\hline 21 & & 2 & 3 & 3'738 & 3'888 & 41 & 125 & 1 & 1 & 0’451 & 0’469 \\
\hline
\end{tabular}


Luego de obtener los valores que corresponden al número de turno óptimo, se simula su comportamiento (Figuras 6 y 7 )

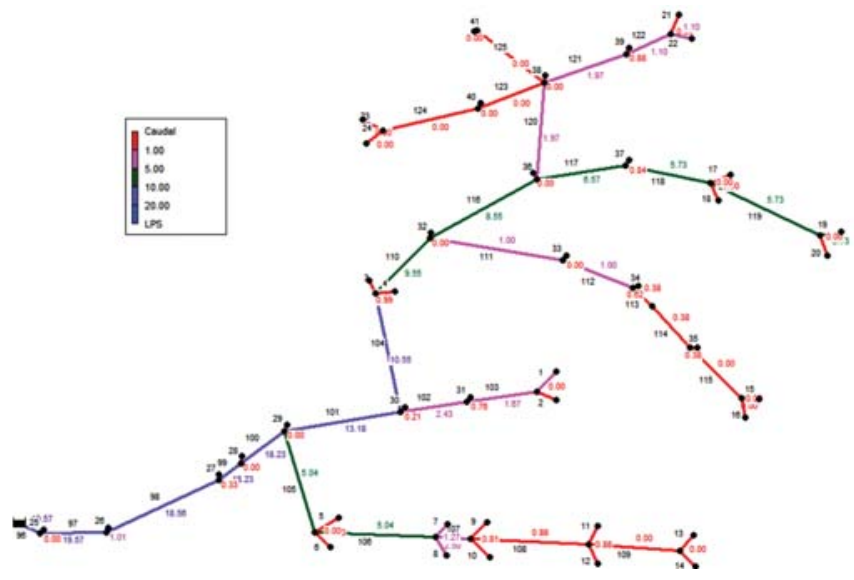

Figura 6. Distribución de caudales al finalizar el primer turno, a la hora 4

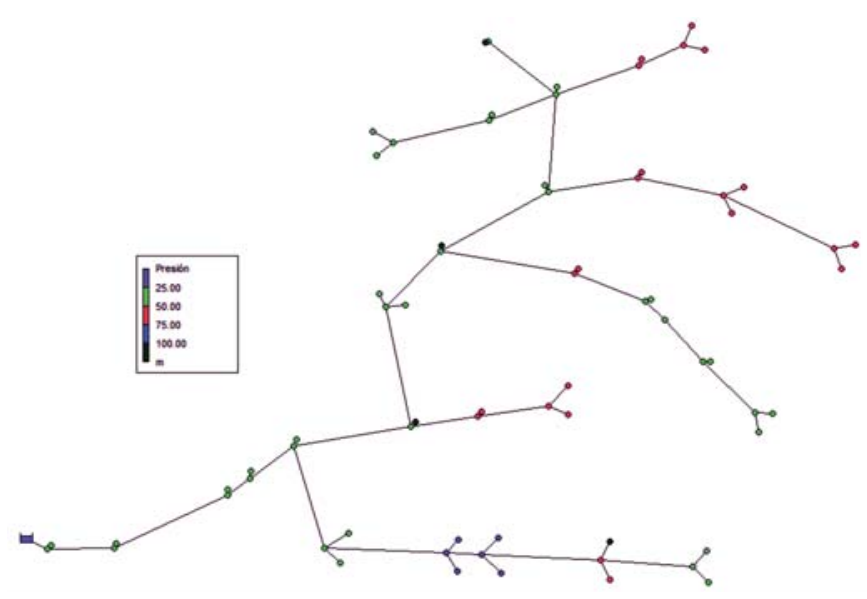

Figura 7. Presión en los nudos de la red a la hora 12
Cuando se aplica el modelo PNLYAG a la red de Tuncarta, con la modalidad de riego por turnos se obtiene un coste total en tuberías que se encuentra valorado en:

\section{Coste Total \\ de la Solución $=\quad 9.033,84$ USD}

\section{Sistema Tres (Cariyacu)}

El sector de la red en el cual se implementó el modelo de PNLYAG está conformado por 49 líneas, 67 hidrantes, y se asumen su funcionamiento con 2 turnos. (Figura 8)

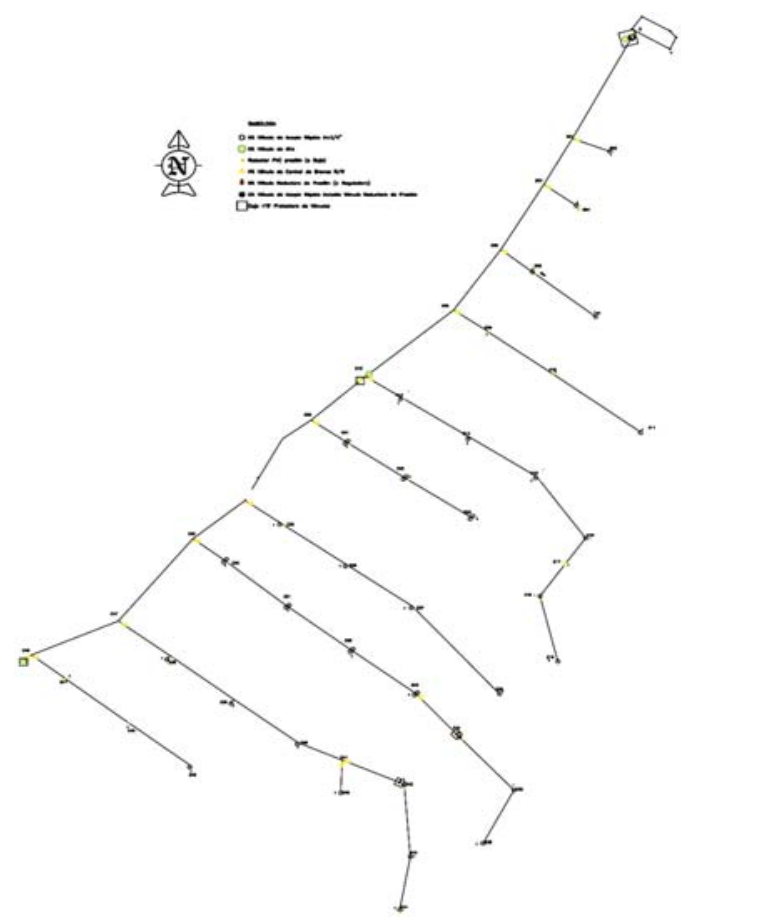

Figura 8. Distribución de caudales

Con la implementación del modelo PNLYAG, la combinación de hidrantes resultante se muestra en la Tabla 11. 
Tabla 11. Combinación de hidrantes con modelo PNLYAG

\begin{tabular}{|c|c|c|c|c|c|c|c|c|c|c|c|}
\hline Nh & Nudo & Hidrante & Turno & Ah (ha) & Qh (I/s) & $\mathrm{Nh}$ & Nudo & Hidrante & Turno & Ah (ha) & Qh (I/s) \\
\hline 1 & 202 & 1 & 2 & 0’3960 & 0’44349 & 35 & 227 & 27 & 1 & 0’5673 & 0'63532 \\
\hline 2 & 204 & 4 & 1 & 0’4318 & 0’48362 & 36 & 227 & 27 & 2 & 0’5673 & 0'63532 \\
\hline 3 & 204 & 4 & 2 & 0’4318 & 0’48362 & 37 & 228 & 28 & 2 & 0’4584 & 0’51341 \\
\hline 4 & 206 & 6 & 1 & 0’3284 & 0’36775 & 38 & 228 & 28 & 2 & 0’4584 & 0’51341 \\
\hline 5 & 206 & 6 & 1 & 0’3284 & 0’36775 & 39 & 230 & 30 & 1 & 0’2668 & 0’29882 \\
\hline 6 & 207 & 7 & 1 & 0'1821 & 0'20390 & 40 & 230 & 30 & 2 & 0’2668 & 0’29882 \\
\hline 7 & 207 & 7 & 1 & 0’1821 & 0’20390 & 41 & 231 & 31 & 1 & 0’2558 & 0’28650 \\
\hline 8 & 209 & 9 & 2 & 0’4125 & 0’46200 & 42 & 231 & 31 & 2 & 0’2558 & $0 ’ 28650$ \\
\hline 9 & 209 & 9 & 1 & 0’4125 & 0’46200 & 43 & 232 & 32 & 2 & 0'2770 & 0’31018 \\
\hline 10 & 210 & 10 & 2 & 0’3520 & 0’39418 & 44 & 232 & 32 & 2 & 0’2770 & 0’31018 \\
\hline 11 & 210 & 10 & 2 & 0’3520 & 0’39418 & 45 & 233 & 33 & 1 & 0’2193 & 0’24562 \\
\hline 12 & 211 & 11 & 1 & 0’2225 & 0’24920 & 46 & 233 & 33 & 1 & 0’2193 & 0’24562 \\
\hline 13 & 211 & 11 & 1 & 0’2225 & 0'24920 & 47 & 235 & 35 & 2 & 0’4582 & 0'51314 \\
\hline 14 & 213 & 13 & 2 & 0’3485 & 0’39026 & 48 & 236 & 36 & 1 & 0’6140 & 0’68768 \\
\hline 15 & 213 & 13 & 1 & 0’3485 & 0’39026 & 49 & 236 & 36 & 1 & 0’6140 & 0’68768 \\
\hline 16 & 214 & 14 & 1 & 0’3657 & 0’40953 & 50 & 238 & 38 & 2 & 0’6671 & 0’74710 \\
\hline 17 & 214 & 14 & 2 & 0'3657 & 0’40953 & 51 & 238 & 38 & 2 & 0’6671 & 0'74710 \\
\hline 18 & 215 & 15 & 2 & 0’3577 & 0’40057 & 52 & 239 & 39 & 2 & 0’4489 & 0'50277 \\
\hline $\begin{array}{l}19 \\
20\end{array}$ & 215 & 15 & $\begin{array}{l}1 \\
2\end{array}$ & $\begin{array}{l}03577 \\
0,5257\end{array}$ & $\begin{array}{l}0,40057 \\
0,5888\end{array}$ & 53 & 239 & 39 & 2 & 0'4489 & 0’50277 \\
\hline 20 & $\begin{array}{l}216 \\
217\end{array}$ & $\begin{array}{l}16 \\
17\end{array}$ & 2 & 0,6540 & $0 ’ 73250$ & 54 & 240 & 40 & 1 & 0’3911 & 0'43798 \\
\hline 22 & 218 & 18 & 2 & 0’7034 & 0’78781 & 55 & 240 & 40 & 1 & 0’3911 & 0’43798 \\
\hline 23 & 219 & 19 & 1 & 0'6053 & 0'67791 & 56 & 242 & 42 & 1 & 0’5982 & 0'67004 \\
\hline 24 & 219 & 19 & 1 & 0’6053 & 0’67791 & 57 & 243 & 43 & 2 & 0’7132 & 0’79877 \\
\hline 25 & 221 & 21 & 1 & 0'2543 & 0’28476 & 58 & 244 & 44 & 2 & 0’3236 & $0 ’ 36238$ \\
\hline 26 & 221 & 21 & 2 & 0’2543 & 0’28476 & 59 & 244 & 44 & 2 & 0’3236 & 0’36238 \\
\hline 27 & 222 & 22 & 1 & 0’2717 & 0’30430 & 60 & 245 & 45 & 1 & 0’5051 & 0’56571 \\
\hline 28 & 222 & 22 & 1 & 0’2717 & 0’30430 & 61 & 245 & 45 & 1 & 0’5051 & 0’56571 \\
\hline 29 & 223 & 23 & 1 & 0’4090 & 0’45802 & 62 & 247 & 47 & 2 & 0’4358 & 0’48804 \\
\hline 30 & 223 & 23 & 2 & 0’4090 & 0’45802 & 63 & 247 & 47 & 1 & 0'4358 & 0’48804 \\
\hline 31 & 225 & 25 & 1 & 0’4916 & 0’55059 & 64 & 248 & 48 & 2 & 0’3498 & 0’39178 \\
\hline 32 & 225 & 25 & 1 & 0’4916 & 0’55059 & 65 & 248 & 48 & 1 & 0’3498 & 0’39178 \\
\hline 33 & 226 & 26 & 1 & 0’4355 & 0’48776 & 66 & 249 & 49 & 2 & 0’3733 & 0'41810 \\
\hline 34 & 226 & 26 & 2 & 0’4355 & 0’48776 & 67 & 249 & 49 & 1 & 0’3733 & 0'41810 \\
\hline
\end{tabular}


Simulando las variables hidráulicas obtenemos la distribución de caudales y presiones (Figura 9 y 10)

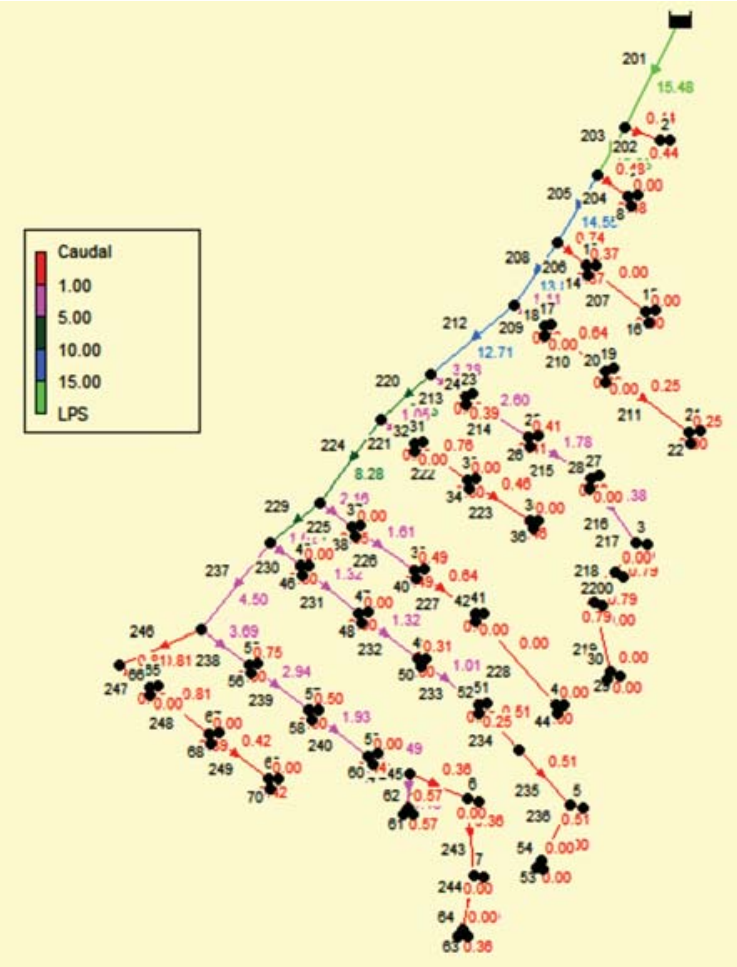

Figura 9. Distribución de caudales a la hora 2.

Cuando se aplica el modelo PNLYAG a la red de Cariyacu con la modalidad de riego por turnos se obtiene un coste total en tuberías que se encuentra valorado en:

\section{Coste Total de la Solución $=\quad 10.130,02$ USD}

Para comprobar el buen funcionamiento de las redes se ha simulado diferentes escenarios como se muestra en las (Figuras 9 y 10) en donde se observa la distribución de caudales en las líneas de la red, y los horizontes de presión de los nudos demandantes

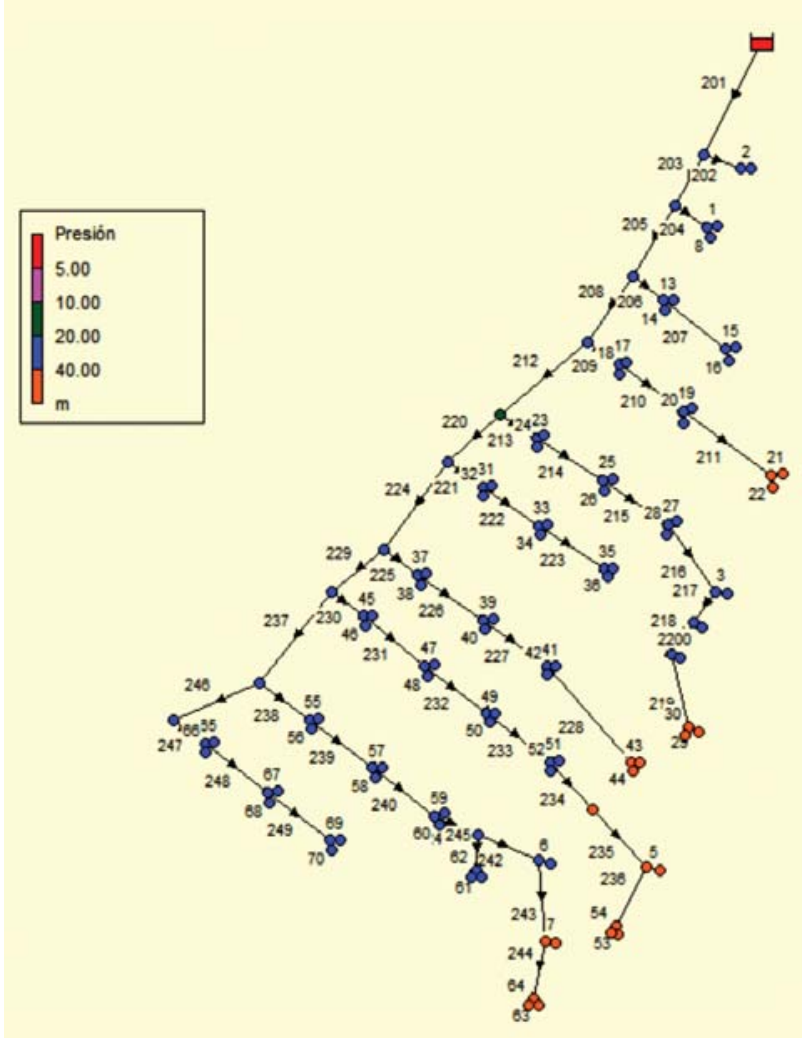

Figura 10. Presión en los nudos de la red a la hora 2.

en las redes en forma respectiva, éstos cumplen con las condiciones de servicio requeridos.

En la Figura 11, se realiza la comparación de los resultados que se obtienen con diseño original a la demanda de la red de Tuncarta, y los resultados de diseño con la metodología desarrollada. Observamos la bondad del método propuesto ya que los diámetros resultantes llegan hasta un dimensionamiento de $150 \mathrm{~mm}$ que implica menor coste, y en el diseño original se obtienen diámetros de hasta $250 \mathrm{~mm}$, lo que se traduce en un coste total correspondiente al $50 \%$ del coste original. 


\section{COMPARACION DISEÑOS}

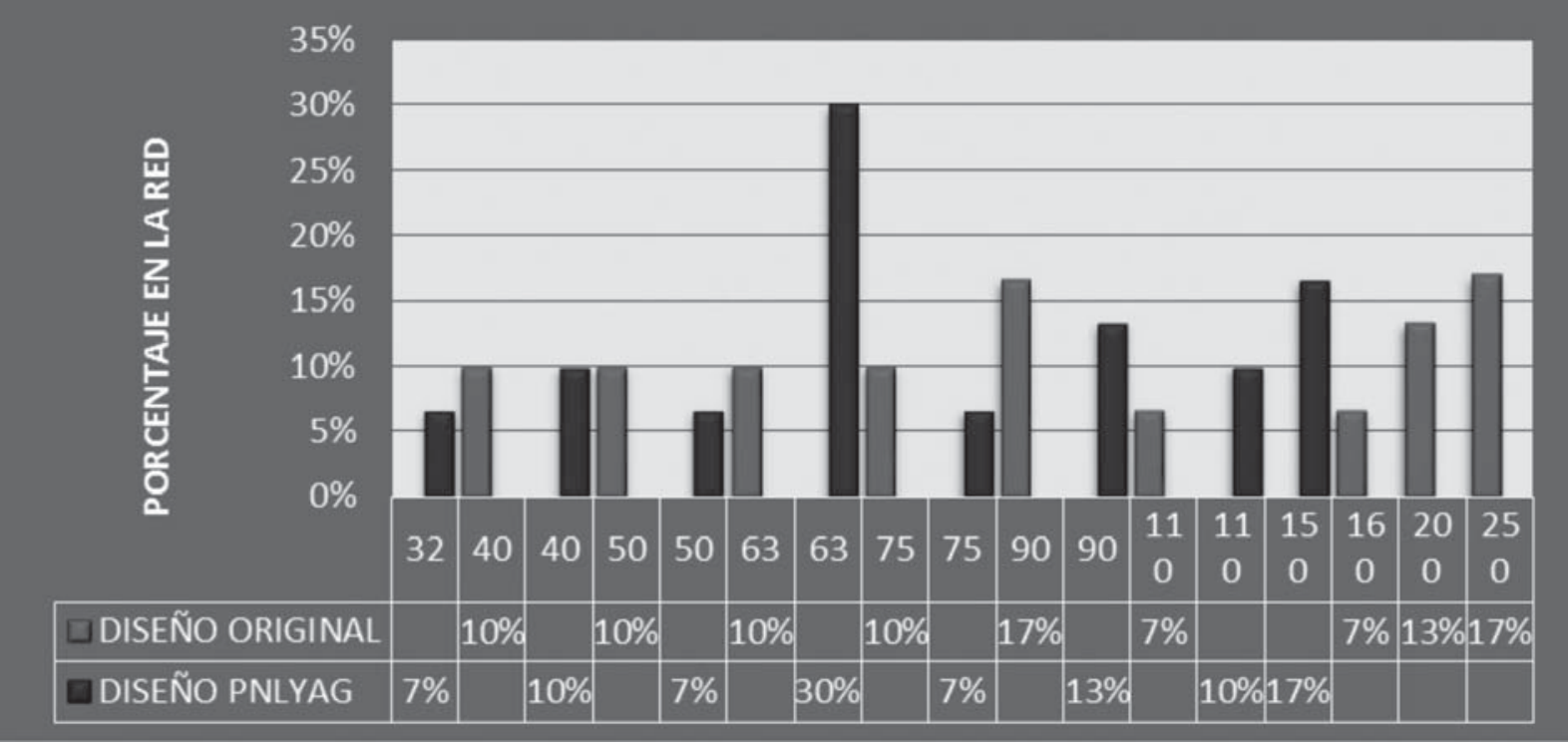

Figura 11. Resultados obtenidos aplicando metodología PNLYAG red Tuncarta.

\section{Conclusiones}

Se han planteado dos metodologías para combinar en forma óptima la asignación de turnos de los hidrantes de redes de riego funcionando en esta modalidad, que permiten obtener el costo mínimo de diámetros de tuberías, la primera fusiona Programación Lineal y Algoritmos Genéticos y la segunda combina Programación No Lineal y Algoritmos Genéticos de tal manera que los resultados además cumplen las prestaciones de servicio en cuanto a presión, caudal y velocidad.

En los casos de estudio en donde se implementaron modelos híbridos, se disminuyó el costo en diámetros diseñados hasta un $50 \%$, con respecto a optar con el diseño de la red a la demanda.

En el modelo híbrido PNLYAG se obtiene un coste total menor debido a la selección de diámetros continuos, lo que no deja de ser un diseño aproximado.

En el modelo PLYAG se obtiene un costo óptimo global que permitirá tomar una decisión de diseño real, ya que usa para su selección diámetros discretos.

\section{Trabajos futuros}

El desarrollo implementado constituye el inicio de la aplicación de Investigación Operativa u Optimización en redes de riego en la región de estudio, con esta herramienta se obtiene una aproximación del diseño de redes que permitirá lograr una mayor efectividad en esta etapa.

Es necesario implicar en el modelo híbrido, sistemas de bombeo; de esta manera también se conocerá la combinación de turnos idónea para obtener diseños óptimos de la red con cada configuración del sistema.

En los modelos propuestos se podría acoplar la automatización de la red y los turnos resultantes, así como posibles alternativas para "flexibilizar" cambios y redistribución de turnos, tanto por necesidades de los regantes como de la propia operación del sistema. 


\section{Agradecimientos}

Se expresa el agradecimiento a la Universidad Politécnica de Valencia, en la persona del Dr. Rafael Pérez quien constituyó una guía eficaz en la ejecución de este trabajo, a la Senescyt (Secretaría de Ciencia y Tecnología de Ecuador) que a través del apoyo económico hizo posible el desarrollo de esta investigación, a la Universidad Técnica Particular de Loja y a todas las personas y entidades que aportaron para la consecución de esta tarea encomendada.

\section{Literatura citada}

1. Alperovits, E. \& Shamir, U. (1977). Design of Optimal Water Distribution Systems, Water Resources Research, 13, (6):885-900.

2. Baños, R. (2006). Meta-heurísticas Híbridas para optimización. Mono-objetivo y Multi-objetivo. Paralelización y Aplicaciones. (Tesis de Doctorado). Universidad de Almería.

3. Bhave, P.R. (1979). Selecting Pipe Sizes in Network Optimization by Linear Programming. Journal of the Hydraulics Division (ASCE), 105(HY7): 1019-25.

4. Calhoun, C.A. (1970). Optimization of Pipe Systems by Linear Programming, in: Proc. of the Institute on Control of Flow in Closed Conduits, Ed. J. P. Tullis, Fort Collins: Colorado State Univerity.

5. Sánchez, R., Laguna, F., Juana L., Losada, A., CastaIlón, G. Rodríguez, L. \& Gil, M. (2009). Organización de turnos para la optimación energética de redes colectivas de riego a presión. (Agua y Energía). Jornadas de Ingeniería del Agua, España. http://oa.upm.es/5718/1/
INVE_MEM_2009_70546.pdf Fecha de acceso: 5 de octubre de 2010.

6. Davies, T.E. \& Príncipe, J.C. (1991). A Simulated Annealing Like Convergence Theory for the Simple Genetic Algorithm. Proceedings of the Fourth International Conference on Genetic Algorithms, San Mateo, California (USA), pp. 174-181.

7. De Jong K.A. (1975). An analysis of the behave our of a class of genetic adaptive systems. Tesis doctoral, University of Michigan.

8. Karmeli, D., Gadish, Y. \& Meyers, S. (1968). Design of Optimal Water Distribution Networks, Journal of the Pipeline Division, ASCE, 94( PL1): 1-10.

9. Labye, Y. (1966). Etude des Procédés de Calcul Ayant PourBut de Rendre Minimal le Cout d' un Reseau de Distribution d' Eau Sous Presion, La Houille Blanche, No 5, Mayo, 577-583.

10. Lamaddalena, N. \& Sagardoy, J.A. (2000). Performance Analysis of on-demand pressurized irrigation systems. FAO Irrigation and drainage. Water Resources. Paper. 59. Roma: Developmet and Management Service FAO Land and of Water Developmet Division.

11. Lara, B. (2007). Diseño de redes colectivas de riego: Estudio de la asignación óptima de caudales mediante algoritmos genéticos. Tesis de Doctorado. Universidad Politécnica de Valencia. España.

12. Mora, D., Iglesias P., Fuertes V. \& Martinez S. (2010). Diseño de redes de agua aplicando técnicas evolutivas. XXIV Congreso Latinoamericano de Hidráulica. Uruguay.

13. Pérez, R. (1993). Dimensionamiento óptimo de redes de distribución de agua en redes ramificadas considerando los elementos de regulación, Tesis de Doctorado, Universidad Politécnica de Valencia, España.

14. Villa, F. (2010). Ubicación óptima de hidrantes multiusuario en redes de riego a presión mediante el empleo de modelos de localización-asignación, Tesis de Máster, Universidad Politécnica de Valencia, España. 